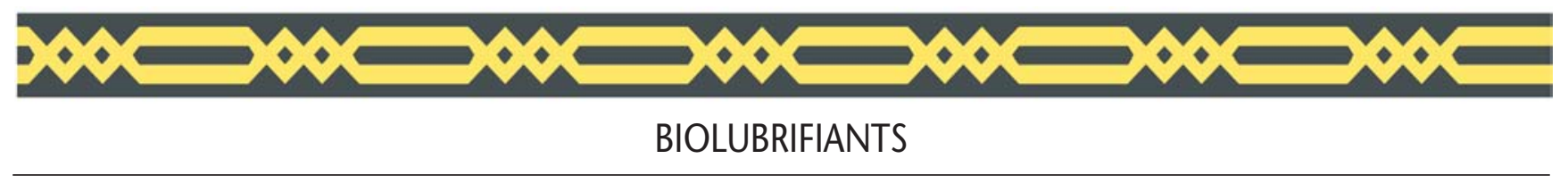

\title{
Lubrifiants et environnement
}

\section{Pascale DE CARO}

\section{Christine CECUTTI}

Laboratoire de chimie agro-industrielle, UMR 1010 INRA/INP- ENSIACET, 118 route de Narbonne, 31077 Toulouse cedex 04

Abstract: As figures show, volumes of lost lubricants in Europe like in most of industrial countries represent a threat for the environment and for the human health. Numerous activity fields are concerned through equipment involved in transports, in construction, in mechanical industry, in forest harvesting or in agriculture... The spread of lubricants in the environment affects different parts of the natural medium (soils, underground waters, rivers...), which explains the difficulty to select analytic methods assessing the real environmental impact of these products. The most usual analyses provides degradation rates of the substance in contact with microorganisms, doses of non-toxicity or measures regarding to the oxygenation rate of waters. It is important to select the most adapted method according to the context. For a few years, vegetable based lubricants have been considered as a reliable solution to combine technical performances and ecocompatibility characteristics. These meet criteria defined in ecolabels or other classifications concerning several categories of biolubricants. National and european programs aiming at technology transfer allowed to measure and compare the ultimate biodegradability in liquid and in soil for hydraulic fluids. The results obtained show an environmental gain for biolubricants included after a 1,000 hours operation period.

Key words: lubricants, environment, biodegradability

chaque année en Europe et avec quelles conséquences pour l'environnement et pour la santé humaine?

que soit le secteur d'application, au de leur cycle de vie, une partie des lubrifiants consommés entre inévitablement en contact avec le milieu environnant. On constate que les lubrifiants dérivés de la pétrochimie présentent des risques pour les écosystèmes et donc pour les ressources naturelles (eau, cultures...) [1]. Outre I'huile de base, les lubrifiants contiennent un certain nombre d'autres substances destinées à améliorer les performances lubrifiantes. Ces composés jouent également un rôle non négligeable au niveau de l'impact environnemental. En effet, les analyses et les contrôles portant sur la nature de ces additifs ne sont pas systématiques, ce qui laisse encore le champ libre à l'utilisation de substances peu compatibles avec l'environnement. Pourtant, il existe des solutions techniques pour faire évoluer les formulations et leur mode d'utilisation. Les formulations d'origine végétale constituent à ce titre une alternative intéressante: un niveau d'écocomptatibilité élevé, une faible inflammabilité et l'utilisation de ressources renouvelables pour ne pas contribuer à l'effet de serre [2].

En Europe, les systèmes de collecte récupèrent environ $30 \%$ des lubrifiants usagés sur les 4,8 millions de tonnes consommées, tandis que $45 \%$ sont considérés comme consommés ou brûlés ${ }^{1}$. Que devient le million de tonnes perdu

\footnotetext{
${ }^{1}$ Source: UEIL (Union européenne des indépendants en lubrifiants).
}

\section{Interaction entre \\ les lubrifiants et l'environnement}

En lubrification perdue, par définition, il n'est pas possible de récupérer le lubrifiant après utilisation. La lubrification perdue représente environ $20 \%$ de la consommation totale en lubrifiants (source CPL 2003).

Un certain nombre d'applications sont concernées :

- les graisses utilisées en système ouvert sont appliquées régulièrement sur les engrenages et mécanismes de divers équipements (écluses, aiguillages de trains, équipements de station de ski...);

- les huiles pour moteur deux temps génèrent dans l'atmosphère des composés de combustion ;

- les agents décoffrants utilisés dans le BTP se retrouvent sous la forme de dépôts sur les moules et sur les parements avant d'être lessivés sur le sol;

- les huiles de chaînes en exploitation forestière et les huiles de coupe utilisées dans les carrières sont dispersées dans des milieux naturels souvent fragilisés :

- les huiles d'ensimage appliquées sur les fils textiles pour les protéger et pour réduire l'élec- tricité statique, se retrouvent dans les eaux de lavage.

La lubrification en système fermé, lors d'une utilisation normale, limite le contact avec l'extérieur, mais il subsiste des pertes accidentelles. Ainsi, la rupture des flexibles sous pression est à l'origine de la perte de 20 à $30 \%$ des fluides hydrauliques et des huiles de transmission. Ces rejets concernent de nombreux secteurs d'activités tels que les travaux publics, I'exploitation forestière, I'agriculture ou l'entretien des cours d'eau... Par ailleurs, $50 \%$ des huiles moteurs ne sont pas comptabilisées dans les circuits de collecte à cause des rejets " sauvages $»[3]$.

Les lubrifiants rejetés dans l'environnement peuvent se retrouver directement au contact de l'eau lorsqu'il s'agit d'équipements partiellement immergés (bateaux, écluses, centrales hydrauliques...) ou bien d'équipements travaillant à proximité des cours d'eau ou du littoral. Or il suffit d'un litre de lubrifiant pour polluer mille litres d'eau potable [4].

En 1997, la pollution du Rhin par les huiles minérales a été estimée à 1032 tonnes par an sur un total de polluants de 5000 tonnes [5].

De façon générale, la dispersion des lubrifiants dans l'environnement affecte les différents compartiments du milieu naturel. Un sol souillé peut donner lieu à une contamination des nappes d'eau souterraines via la migration des produits au travers du sol et conduire à la pollution des eaux de rivières par les eaux de 
ruissellement qui emportent avec elles les agents polluants.

De la pollution environnementale, découle un impact sanitaire ; c'est la qualité des eaux qui est en question et, plus ponctuellement, ce sont les cultures qui peuvent être contaminées. Ce constat permet d'identifier les lubrifiants comme une source de pollution non négligeable mais qui s'opère de façon insidieuse du fait du fractionnement des déperditions sur I'ensemble des territoires.

\section{Évaluation de l'impact environnemental des lubrifiants}

Pour cette évaluation, il est préférable de prendre en compte l'impact de l'ensemble de la formulation (huile de base et additifs). II est également plus représentatif de réaliser des mesures sur le lubrifiant usagé. En effet, on peut craindre une baisse de la qualité environnementale lorsque le lubrifiant $d^{\prime}$ 'un système clos se charge en métaux d'usure (principalement du fer, du cuivre et du plomb) ou lorsqu'il a subi une dégradation chimique du fait d'une utilisation à hautes températures.

L'impact des lubrifiants sur le milieu environnant nécessite de prendre en compte plusieurs paramètres présentés ci-après.

Ainsi, il faut regarder de quelle façon se décomposent les substances au contact des microorganismes présents dans le milieu naturel ; il s'agit de mesurer la biodégradabilité de la formulation.

II faut également caractériser l'impact des substances sur le développement et la survie des organismes vivants; il s'agit de mesures d'écotoxicité.

Il existe d'autres types de mesures pour évaluer le degré de pollution du milieu et qui apportent des renseignements complémentaires. Par exemple, pour les eaux, on peut déterminer la $D B O$ (demande biologique en oxygène) et la DCO (demande chimique en oxygène) et pour les sols, le coefficient de partage octanol/eau (W/O) qui rend compte de l'affinité des polluants avec l'eau et donc de leur persistance dans le sol [6]. Outre la détermination de ces paramètres, l'impact environnemental d'une substance doit normalement prendre en compte le paramètre de migration au travers des différentes couches du sol : un suivi spatio-temporel permet de contrôler la dégradation du polluant dans le temps et dans I'espace $[7,8]$. Une migration trop rapide du produit avec les eaux de drainage conduit à une pollution des eaux souterraines. Au contraire, si le produit reste adsorbé sur les particules de sol, dans la mesure où il est biodégradable, il peut être transformé par les micro-organismes et ne causera pas de dégâts environnementaux.

\section{La biodégradabilité}

Plus les processus naturels de la biodégradation sont rapides et moins la substance est polluante envers le milieu environnant. La biodégradabilité s'exprime donc en pourcentage de substance dégradée durant un temps déterminé, généralement 28 jours. Pour être biodégradés, les hydrocarbures doivent passer par une étape supplémentaire d'oxydation par rapport aux esters ou aux acides gras qui, eux, sont directement accessibles par les micro-organismes via la fonction carboxylique.

Le taux de biodégradation peut être évalué en suivant la disparition du composé parent : il s'agit-là de la biodégradabilité primaire qui se base sur le test CEC L33T94 réalisé en milieu aqueux. C'est la méthode la plus simple à mettre en œuvre et, de ce fait, la plus utilisée par les formulateurs. Mais cette méthode, de par son principe, ne traduit pas réellement l'impact environnemental des substances étudiées car elle ne se préoccupe pas du devenir des métabolites formés lors de la décomposition du composé parent. Or, il peut y avoir un grave danger environnemental au niveau de la toxicité et de la persistance de ces métabolites. Ainsi, les taux mesurés à l'aide de cette méthode sont-ils souvent supérieurs à ceux donnés par la biodégradabilité ultime. Cette dernière est déterminée via la quantité de dioxyde de carbone émis en stade final des processus de dégradation du composé parent. Précisément, on détermine la quantité de matière qui est transformée en éléments chimiques inoffensifs pour l'environnement (dioxyde de carbone, eau, biomasse). Le test OCDE 301B basé sur ce principe est le test de référence. Il est réalisé en milieu aqueux avec des micro-organismes provenant de boues de stations d'épuration.

Il est généralement admis qu'un produit est facilement biodégradable quand son taux de biodégradabilité dépasse $60 \%$ au bout de 28 jours, selon la ligne directrice OCDE 301. Le critère de la fenêtre des 10 jours stipule que le fluide doit atteindre les $60 \%$ de dégradation sur une période de 10 jours, commençant lorsque le taux de biodégradation atteint $10 \%$ et finissant avant le $28^{\mathrm{e}}$ jour de l'essai.

Les protocoles de biodégradabilité (OCDE et CEC) sont basés sur des mesures en milieu aqueux. Or, de nombreux lubrifiants sont d'abord dispersés sur le sol. Ces protocoles ne sont pas alors forcément adaptés. En effet, le sol est moins bien oxygéné que l'eau, les microorganismes contenus dans le sol sont différents de ceux que l'on peut trouver en milieu aqueux, et enfin, l'adsorption des substrats aux particules du sol diminue leur accessibilité visà-vis des micro-organismes. La biodégradabilité ultime peut également être évaluée dans le sol à l'aide d'une nouvelle méthode qui a fait
I'objet d'un préprojet de norme Afnor NF X31222 [9]. Cette méthode, basée sur le principe du test OCDE 301 B, permet d'évaluer la biodégradabilité ultime de substances déposées dans un bioréacteur contenant du sol naturel avec les micro-organismes d'origine, par dosage du dioxyde de carbone formé au cours des processus de biodégradation.

\section{L'écotoxicité}

Elle est mesurée au moyen de tests normés qui mettent en œuvre des organismes représentatifs de la chaîne alimentaire. Les tests d'écotoxicité qui font référence sont les lignes directrices OCDE 201, 202 et 203 qui représentent le milieu aquatique à travers les algues, les daphnies et les poissons. On mesure les doses seuils qui provoquent chez ces êtres vivants des perturbations dans leur comportement (inhibition de croissance pour les algues, immobilisation pour les daphnies) ou leur mort (dose létale pour les poissons). Plus les seuils d'écotoxicité sont bas, plus les produits testés sont toxiques. Si on se réfère aux phrases de risque [10], on considère un composé comme étant toxique quand il cause un effet négatif envers les organismes vivants à une teneur inférieur à $100 \mathrm{mg}$ par litre.

II faut noter que la biodégradabilité et l'écotoxicité sont deux paramètres indépendants et donc deux conditions à remplir simultanément pour répondre au critère d'écocompatibilité

\section{Pollution des eaux}

La mesure de la DBO et DCO est une méthode générale pour évaluer le degré de pollution d'une eau par les matières organiques. Elle requiert une bonne dispersion du lubrifiant dans le prélèvement aqueux.

Les rejets de lubrifiants en milieu aqueux sont également concernés par la norme NF EN ISO 9377-2 qui porte sur le dosage des hydrocarbures totaux conformément à la réglementation sur la qualité des eaux, relative aux installations classées (arrêté du 2 février 1998). Cette norme vient se substituer à la norme française NF T90-114 dont le protocole de dosage présentait l'inconvénient d'assimiler une partie des esters à des hydrocarbures [11]. Dans ces conditions, il est facile de faire valoir le bénéfice environnemental lié à l'utilisation d'une formulation sur base d'ester végétal.

II s'agit d'un exemple pour lequel le processus de substitution doit s'accompagner d'une évolution des méthodes d'analyse. 


\section{Caractéristiques environnementales des biolubrifiants issus de ressources renouvelables}

Depuis une dizaine d'années, un certain nombre d'organismes et de réseaux professionnels se sont mobilisés avec la collaboration de quelques formulateurs pour mettre au point des lubrifiants plus respectueux de l'environnement et pour favoriser leur utilisation. Les différents programmes consacrés à ces études montrent que dans de nombreux cas l'utilisation de lubrifiants d'origine naturelle est une solution fiable pour combiner performances techniques et compatibilité avec l'environnement [12-14].

Dans certains cas, la substitution d'un lubrifiant d'origine minérale par un lubrifiant d'origine végétale s'accompagne d'une modification du protocole d'application, ce qui du même coup favorise la mise en place de pratiques plus respectueuses de l'environnement.

On peut citer le cas du travail des métaux, pour lequel la technique de micropulvérisation de fluide végétal diminue considérablement les volumes de lubrifiants consommés et donc le stockage des rejets. Concernant les huiles de décoffrage, il a été montré que l'utilisation de formulations végétales doit s'accompagner $d^{\prime} u n e$ réduction des doses d'emploi pour optimiser les performances de démoulage [15]. Le développement d'émulsions aqueuses est un autre moyen de réduire le rejet de matières actives.

\section{Les référentiels}

Dans certains pays européens comme l'Allemagne, on a pu observer que la mise en place d'écolabels crée un contexte favorable à l'utilisation de lubrifiants respectueux de l'environnement : en répondant à une liste de critères environnementaux associés à quelques spécifications techniques, les formulations lubrifiantes labélisées offrent une garantie de qualité qui rassure les applicateurs.

En France, il existe depuis décembre 2002 un écolabel français de la marque NF-environnement certifié par l'Afnor, qui s'applique aux lubrifiants pour chaînes de tronçonneuse.

Le 9 décembre 2004, les instances européennes ont adopté un écolabel européen pour les lubrifiants, ce qui évite aux formulateurs qui exportent leurs produits de demander une labellisation dans chaque pays. Les produits labellisés sont reconnaissables par le symbole de la fleur reprenant les étoiles du drapeau européen.

Concernant I'industrie textile, il existe également depuis 2002, un écolabel européen qui prend en compte les impacts environnemen- taux et sanitaires sur l'ensemble de la chaîne de production de la fibre. La limitation de I'utilisation de substances nocives pour l'eau, l'air et la santé concerne, entre autres, $90 \%$ des produits de cardage et d'ensimage mis en œuvre. Devant la demande croissante des utilisateurs et des organismes de santé, les adhérents du Synad (Syndicat national des adjuvants pour bétons et mortiers) ont construit une classification des agents de démoulage sur des critères d'information en matière de santé, d'environnement et d'inflammabilité. Les produits adhérents sont identifiés par un logo spécifique. Une appellation végétale a même été créée : elle concerne les agents formulés entièrement ou en partie avec des constituants végétaux et répondant à un seuil minimal de biodégradabilité ultime.

\section{Des campagnes de démonstration sur sites pilotes}

Des programmes de transfert de technologie visant à promouvoir l'utilisation de biolubrifiants sur des sites pilotes, à l'échelle régionale, nationale et européenne $[5,13,16]$ ont permis de démontrer les qualités environnementales des biolubrifiants.

Notamment, des analyses ont été réalisées à partir des fluides hydrauliques qui représentent des volumes importants (600 000 tonnes par an dans l'Europe des quinze). Des prélèvements ont été effectués sur des équipements fonctionnant en conditions normales avec des fluides à base d'esters oléochimiques: pelles mécaniques, bateaux dragueurs et écluses automatisées appartenant aux VNF (voies navigables de France), ainsi que des abatteuses et des porteurs utilisés en exploitation forestière. Les études réalisées sur les fluides usagés après 1000 heures d'utilisation permettent de s'assurer qu'il n'y a pas eu de dégradation de la qualité environnementale des fluides: pas d'enrichissement en métaux d'usure (principa- lement du fer, du cuivre ou du plomb) et absence de modifications chimiques (oxydation, hydrolyse) découlant de leur utilisation à hautes températures et à hygrométrie élevée. Ce suivi analytique a également comporté des mesures de biodégradabilité et d'écotoxicité dont les résultats sont présentés ci-après.

\section{Biodégradabilité ultime en milieu aqueux et en milieu sol}

Le tableau 1 rassemble des résultats de biodégradabilité ultime mesurés sur des biolubrifiants employés comme fluides hydrauliques.

À la lecture de ce tableau, on observe les taux élevés de biodégradabilité ultime mesurés en milieu aqueux pour des biolubrifiants utilisés dans des conditions habituelles de travail pendant au moins 1000 heures. Ces résultats sont intéressants car ils démontrent que les fluides, malgré des conditions drastiques (températures et taux d'humidité élevés), n'ont pas subi d'altération pouvant influer sur leur comportement environnemental. Ce résultat va dans le même sens que la quantification des métaux d'usure et des particules présentes dans les fluides usagés; les teneurs se situent en dessous des seuils limites fixés pour garantir les bonnes propriétés lubrifiantes.

Des études comparatives menées à la fois sur certains de ces biolubrifiants (lubrifiants 1, 2 et 3 ) et sur un lubrifiant traditionnel d'origine pétrolière testé sur porteur, ont montré des écarts notables de taux de biodégradation ultime entre les deux catégories de lubrifiants lorsque l'expérimentation est menée en milieu sol, toujours après 1000 heures d'utilisation (tableau 2). De plus, quel que soit le sol, les taux de biodégradabilité sont inférieurs à ceux mesurés en milieu aqueux; on explique cette tendance à la surévaluation des résultats de biodégradabilité en milieu liquide, par la nature même du milieu qui présente à la fois une meilleure disponibilité du substrat et une

Tableau 1. Biodégradabilité ultime des biolubrifiants usagés mesurée dans l'eau selon la ligne directrice OCDE 301B.

\begin{tabular}{|c|c|c|c|}
\hline Lubrifiant et machine & $\begin{array}{l}\text { Nature du } \\
\text { lubrifiant }\end{array}$ & $\begin{array}{l}\text { Nombre d'heures } \\
\text { d'utilisation }\end{array}$ & $\begin{array}{c}\% \text { de biodégradabilité } \\
\text { ultime }\end{array}$ \\
\hline $\begin{array}{l}\text { Biolubrifiant } 1 \\
\text { sur abatteuse }\end{array}$ & Ester de TMPa & 1000 & 75 \\
\hline $\begin{array}{l}\text { Biolubrifiant } 2 \\
\text { sur porteur }\end{array}$ & 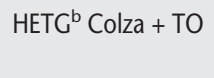 & 1000 & 79 \\
\hline $\begin{array}{l}\text { Biolubrifiant } 3 \\
\text { sur porteur }\end{array}$ & HETC ${ }^{b}$ TO & 1000 & 69 \\
\hline $\begin{array}{l}\text { Biolubrifiant } 4 \\
\text { sur bateau-drague }\end{array}$ & HEES ${ }^{c}$ saturé colza & 2000 & 80 \\
\hline $\begin{array}{l}\text { Biolubrifiant } 5 \\
\text { sur pelle mécanique }\end{array}$ & HEES ${ }^{c}$ saturé colza & 1000 & 85 \\
\hline
\end{tabular}

a TMP : triméthylolpropane.

${ }^{\mathrm{b}}$ HETG : huile environnement triglycéride sur base colza et/ou tournesol oléique (TO).

${ }^{c}$ HEES : huile environnement ester synthétique. 
Tableau 2. Biodégradabilité ultime des lubrifiants usagés mesurée dans deux sols selon le pré-projet de norme Afnor NF X31-222.

\begin{tabular}{|lcc|}
\hline & $\begin{array}{c}\text { Biodégradabilité ultime } \\
\text { en sol sableux (\%) }\end{array}$ & $\begin{array}{c}\text { Biodégradabilité ultime } \\
\text { en sol argileux (\%) }\end{array}$ \\
\hline Biolubrifiant 1 & 60 & 61 \\
Biolubrifiant 2 & 61 & 62 \\
Biolubrifiant 3 & 57 & 57 \\
Lubrifiant traditionnel & 25 & 27 \\
\hline
\end{tabular}

meilleure oxygénation, favorisant ainsi les processus aérobies de la biodégradation. Cependant, la méthode en milieu sol est plus sensible et permet de se rapprocher des conditions réelles de risque de pollution, lorsqu'un lubrifiant est répandu sur le sol de façon accidentelle. On peut ainsi mieux évaluer les risques de pollution des eaux souterraines et du sol, tout en différenciant nettement les lubrifiants selon leur capacité à être dégradé par les microorganismes présents dans le milieu.

\section{Mesures d'écotoxicité}

Des mesures de seuils de toxicité envers des organismes vivants (algues, poissons et daphnies) pratiquées sur les biolubrifiants 1, 2 et 3 vont dans le même sens.

Les résultats rassemblés dans la figure 1 montrent que le lubrifiant d'origine minérale, qu'il soit neuf ou usagé, est toxique à des doses nettement plus faibles que pour les biolubri- fiants, et ceci pour tous les organismes étudiés. On remarque également que la toxicité augmente avec l'usure des fluides, mais cette détérioration reste moindre pour les biolubrifiants, excepté pour le biolubrifiant 3 testé sur les algues : dans ce cas, le seuil de toxicité est assez bas (de l'ordre de 100). On peut imputer cette valeur à la présence de sélénium, élément apporté par les additifs et très toxique envers les êtres vivants. Par ailleurs, il semble que le test sur les algues soit le plus sensible. On voit donc que pour bien évaluer l'écotoxicité des produits, il est préférable de disposer de I'ensemble des trois tests car l'interprétation d'un seul peut conduire à des conclusions erronées.

\section{Résultats de mesures de pollutions aqueuses}

Les mesures ont été réalisées sur des effluents aqueux chargés en huiles d'ensimage à des concentrations qui peuvent correspondre à celles mesurées en sortie d'usine si l'on ne prend pas la précaution de réaliser une dilution avant rejet.

De par la faible solubilité des lubrifiants dans l'eau, les méthodes d'analyse ne sont pas toujours adaptées pour faire la distinction entre les deux types de lubrifiants (origine pétrochimique et végétale). La tendance observée sur la figure 2 dans le cas de l'étude réalisée est favorable [11] à la formulation à base d'ester végétal. Cette dernière permet d'abaisser la DCO ainsi que la concentration «apparente » en hydrocarbure.

\section{Conclusion}

Les différentes données environnementales collectées s'accordent pour faire valoir un gain environnemental positif lié à l'utilisation des biolubrifiants. Les mesures de biodégradabilité ultime et de seuil d'écotoxicité constituent les paramètres de référence en la matière, mais l'interprétation des résultats doit tenir compte des protocoles de mesure mis en œuvre. Par exemple, une mesure de biodégradabilité primaire en milieu aqueux constitue des conditions favorables à la dégradation des composés. La biodégradabilité ultime, plus proche de la réalité donnera des niveaux de biodégradabilité moins élevés et, là encore, on notera un écart entre les mesures réalisées en milieu aqueux ou en milieu sol, ce dernier étant plus sensible pour différencier des niveaux de bio-

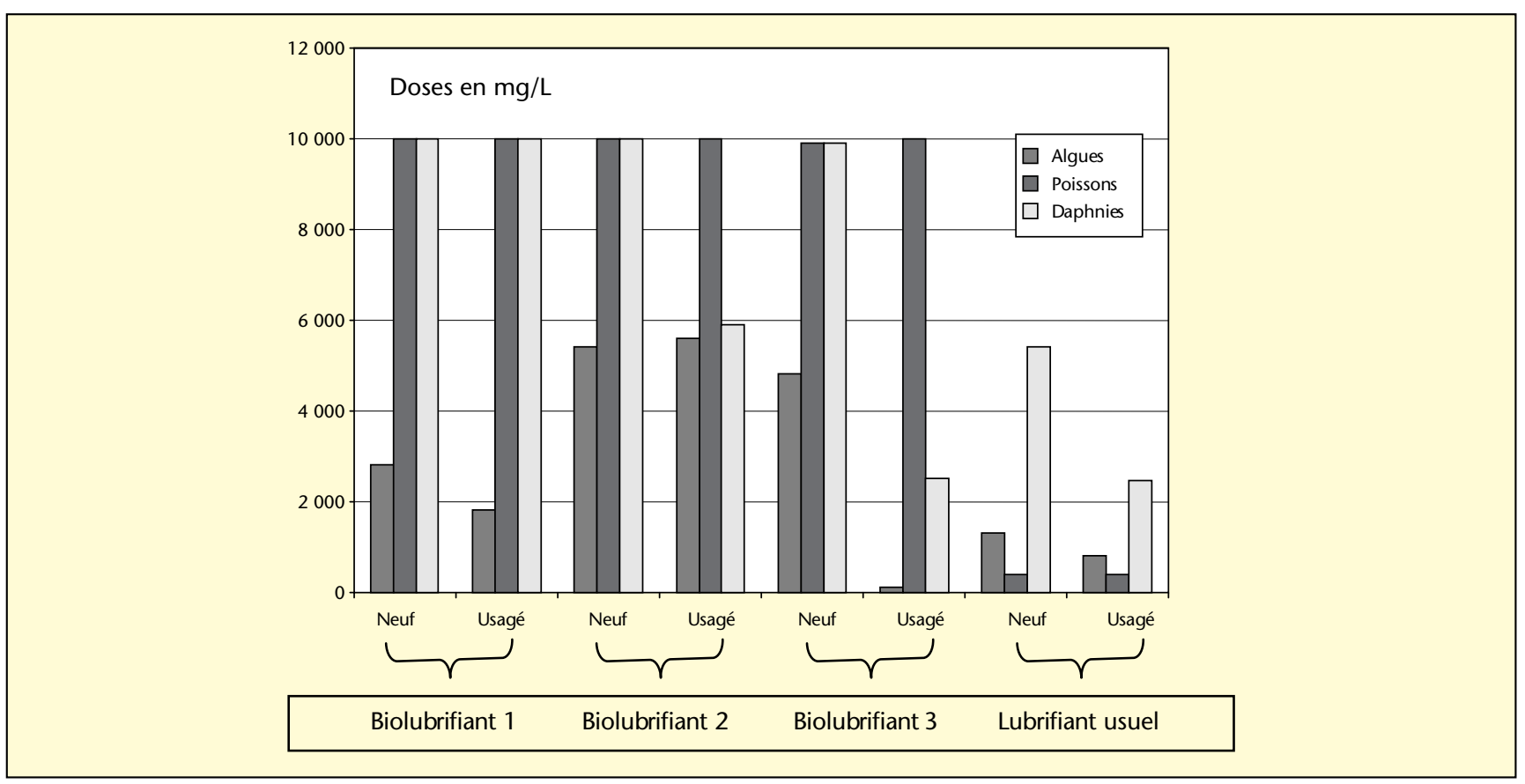

Figure 1. Seuils d'écotoxicité des lubrifiants neufs et usagés. La dose en mg par litre correspond à : une concentration efficace (EC50-72 h) pour les algues selon OCDE 201 ; une concentration létale (LC50-48 h) pour les poissons selon OCDE 203; une concentration efficace (EC50-48 h) pour les daphnies selon OCDE 202. 


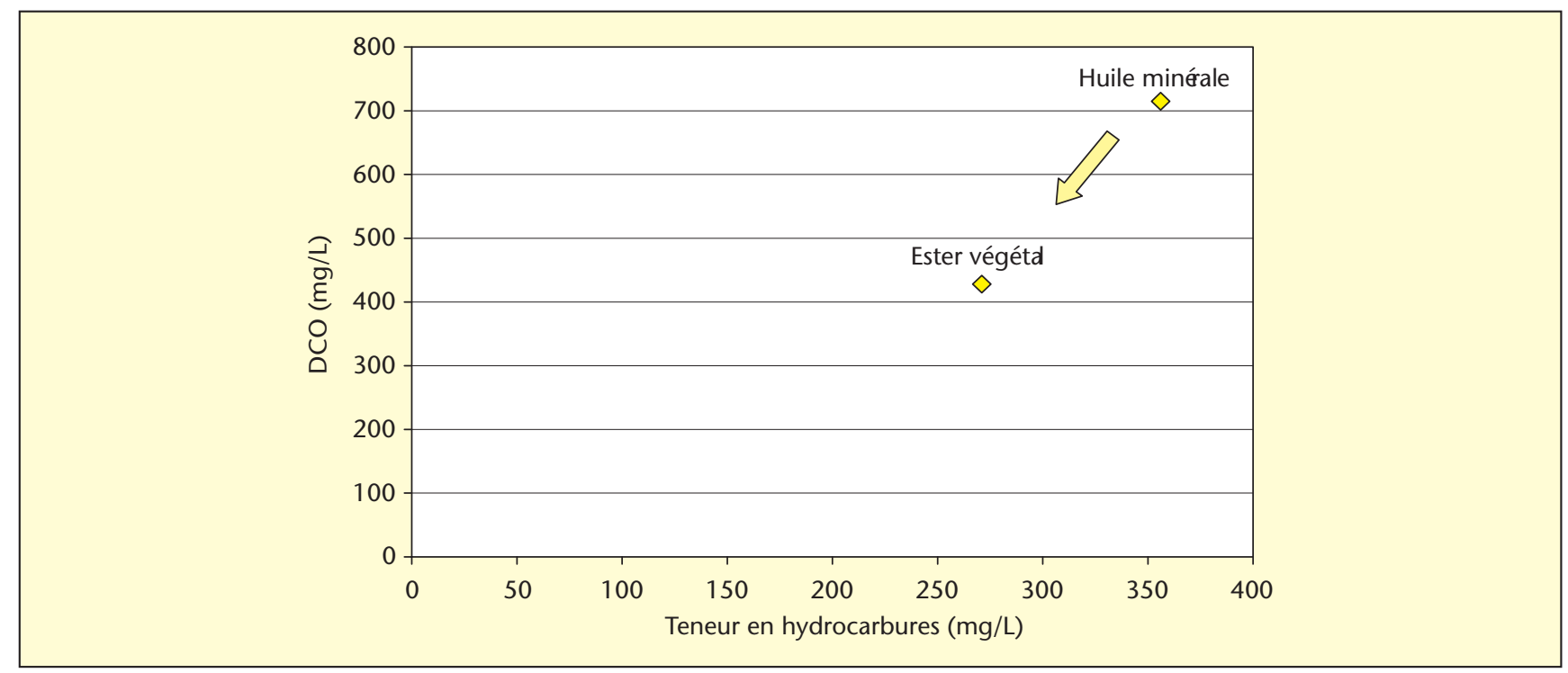

Figure 2. Mesure de la demande chimique en oxygène et détermination de la teneur en HC selon la norme NF T90-114 sur des effluents aqueux reconstitués contenant 200 mg/L (échantillons pour DCO) et $400 \mathrm{mg} / \mathrm{L}$ (échantillons pour HC) d'huiles d'ensimage d'origine pétrochimique et d'origine végétale.

dégradation. De même, afin d'avoir des résultats représentatifs en matière d'écotoxicité, il est conseillé de réaliser les tests sur plusieurs organismes vivants.

Les bilans environnementaux réalisés dans ces programmes de recherche montrent que l'utilisation de biolubrifiants semble être une excellente opportunité pour participer activement à la protection de l'environnement car ces produits grâce à leur caractère facilement biodégradable ne présentent pas de risque de pollution des sols ou des eaux souterraines et les tests écotoxicologiques de référence montrent qu'ils ne sont pas nocifs envers les organismes environnants.

Pour quantifier l'ensemble du gain environnemental lié à la production et à l'utilisation de biolubrifiants, une analyse de cycle de vie serait nécessaire. Les travaux menés dans ce domaine font état généralement d'un bilan positif pour les lubrifiants d'origine naturelle $[17,18]$, mais les résultats peuvent varier sensiblement en fonction du domaine d'application et des données disponibles intégrées dans le bilan. En attendant, il est souhaitable que les biolubrifiants soient explicitement pris en compte dans les descriptifs des systèmes de management environnemental (plans environnementaux, norme ISO 14000) de façon à promouvoir leur utilisation, plus particulièrement en zones sensibles.

\section{RÉFÉRENCES}

1. BARTZ WJ. Lubricants and the environment. New Dir Tribol, Plenary Invited Pap World Tribol Congr, 1st $1997:$ 103-19.
2. WILLING A. Oleochemical esters - environmentally compatible raw material for oils and lubricants from renewable resources. Fett/lipids $1999 ; 101$ : 192-8.

3. DE CARO P. Analyse de la situation relative à I'utilisation de lubrifiant d'origine naturelle en France et dans quelques pays européens, Rapport de la convention de recherche ADEME/ONIDOL nº 9701 007, Novembre 1997.

4. CORNET J. Économie, écologie, lipochimie. OCL 1994 ; 1(2).

5. Lubrication in Inland and Coastal water activities, Rapport final du programme européen LLINCWA, Programme du 5e PCRD «Innovation and SMEs». A. A. Balkema publishers, 2003.

6. WILLING A. Lubricants based on renewable resources - an environmentally compatible alternative to mineral oil products. Chemosphere $2001 ; 43$ : 89-98.

7. CECUTTI C, AGIUS D, CAUSSADE B, GASET A. Fate in the soil of an oil additive of plant origin. Pest Manag Sci 2002 ; 58 : 1236-42.

8. AGIUS D. Biodégradabilité et devenir d'une huile adjuvante d'origine végétale dans le sol. Thèse de doctorat $\mathrm{N}^{\circ} 1669$ de I'INPT, mai 2000.

9. CECUTTI C. Un nouveau test d'évaluation de la biodégradabilité dans le sol-Travaux de normalisation en cours. OCL 2003 ; 10(5-6) : 354459.

10. Directive européenne sur la classification des substances dangereuses 67/548/CEE ( $9^{\mathrm{e}}$ amendement).
11. DE CARO P. Évaluation des performances des huiles d'ensimage textile formulées sur base d'esters - développement de méthodes analytiques adaptées. Rapport ADEME, février 2002.

12. DE CARO P, NGUYEN THE N. Vegetable based lubricants in Forestry: results from a test campain. Agro-Food-Industry High-Tech $2001 ; 4$ : 26-9.

13. DE CARO P. Mise en place et suivi d'un réseau de démonstration pour l'utilisation de biolubrifiants : application au secteur de la viticulture, Rapport ADEME, septembre 2003.

14. MARTINS R, SEABRA J, BRITO A, SEYFERT C, LUTHER R, IGARTUA A. Friction coefficient in FZG gears lubricated with industrial gear oils: biodegradable ester vs. mineral oil. Tribology International (2005, A paraître).

15. DE CARO P. Utilisation d'huiles à base végétale pour le démoulage de matériaux de construction. Rapport ADEME, septembre 2000.

16. CECUTTI C. Impact environnemental de lubrifiants d'origine végétale utilisés dans l'exploitation forestière. Programme AGRICE de la convention de recherche $n^{\circ} 0001013$, juin 2001.

17. WIGHTMAN PS. Environmental benefits to be derived from the use of vegetable oils in place of existing petrochemical materials. CTVO-net Workshop, november 1998.

18. HEKSTER F. A comparative study to the environmental effects of mineral oil and vegetable oil based lubricants in open lubricating systems, Report for LLINCWA Program, Chemiewinkel- Université d'Amsterdam, avril 2001. 